\title{
MOISTURE COMFORT AND ANTIBACTERIAL PROPERTIES OF ELASTIC WARP-KNITTED FABRICS
}

\author{
Zhi-Cai Yu' ${ }^{1}$, Jian-Fei Zhang ${ }^{2}$, Ching-Wen Lou ${ }^{3}$, Hua-Ling He ${ }^{1}$, An-Pang Chen ${ }^{4}$, and Jia-Horng Lin ${ }^{4,5,6 *}$ \\ ${ }^{1}$ Eastern Liaoning University, Functional Textile Materials Laboratory of Eastern Liaoning University, \\ School of Chemical Engineering and Material Science, Dandong, 118003, China. \\ ${ }^{2}$ Tianjin Polytechnic University, School of Textiles, Tianjin 300387, China \\ ${ }^{3}$ Central Taiwan University of Science and Technology, Institute of Biomedical Engineering and Materials Science, Taichung, 40601, Taiwan \\ ${ }^{4}$ Feng Chia University, Laboratory of Fiber Application and Manufacturing, Department of Fiber and Composite Materials, Taichung, 40724, Taiwan \\ ${ }^{5}$ China Medical University, School of Chinese Medicine, Taichung 40402, Taiwan \\ ${ }^{6}$ Asia University, Department of Fashion Design, Taichung 41354, Taiwan \\ *E-mail: jhlin@fcu.edu.tw
}

\section{Abstract:}

Multifunction elastic warp-knitted fabrics were fabricated on a crochet machine with the use of metal composite yarns/viscose yarn and bamboo polyester/ crisscross-section polyester hybrid yarns as the front face and back face of the knitted fabric structure, respectively. We investigated the effect of the blend ratio of bamboo charcoall crisscross-section polyester multiply yarns on the fabric's moisture comfort properties, such as water vapour transmission (WVT), water evaporation rate (WER), and water absorbency. The results showed that blending ratio significantly influenced WVT and WER. Moreover, antibacterial activity of the elastic warp-knitted fabric was tested against Staphylococcus aureus and Escherichia coli in accordance with AATCC 90-2011. Finally, the extensionstress value curves were used to analyse the elastic stretching property, and the fabric exhibited greater breaking elongation and lower stress value in the walewise than in the weft direction.

\section{Keywords:}

Elastic warp-knitted fabric, heat-moisture comfort property, antibacterial activity, stress value, extension

\section{Introduction}

Comfort is a pleasant, physiological, and psychological state of the human body that can be classified into three groups, namely, psychological, tactile, and heat-moisture comfort [12]. Clothing comfort, particularly the feeling of coolness during the summer season or when engaging in sports activities, is of paramount importance for wearers. As the human body generates heat more quickly during vigorous activities, the moisture transport and water vapour transmission (WVT) properties of a garment highly influence its capability to dissipate the extra heat or water vapour from the human body to the outer environment and consequently maintain the body temperature at $37^{\circ} \mathrm{C}$ through perspiration evaporation [3-6].

In addition to WVT and moisture transport ability, the drying behavior of a garment is also a major factor affecting clothing comfort, because the evaporation of absorbed sweat from the fabric surface is the primary mechanism behind the drying of a garment during wear. The drying behavior of a garment can cool down the body, because some energy would be taken out from the human body through sweat evaporation. A high drying ability of clothing not only provides a desired level of coolness comfort but also decreases the weight from the absorbed sweat [7-9].

Numerous studies have investigated the water vapour or drying behavior of fabrics. Duru et al. [10] investigated the effect of repeated laundering on the drying properties of fabrics and found that the laundering process influenced drying property.
Bedek et al. [11] used inverted cup test method to evaluate the WVT of knitted fabric and found that air permeability significantly influenced WVT.

Although many researchers have investigated the influence of moisture on knitted fabrics, limited research effort has been exerted on the drying behavior, water vapour permeability, and antibacterial activity of elastic warp-knitted fabric. Compared with ordinary knitted fabric, elastic warp-knitted fabric exhibits lower resistance to clothing stretch caused by ordinary body movement [12]. When clothing is worn during sports activities or at high ambient temperature, the adsorbed perspiration encourages the growth of microorganisms in the clothing, thus causing several health risks. Such bacterial reproduction can be avoided by the addition of an antibacterial finishing agent and the incorporation of antibacterial yarns into the fabric.

In this study, a type of multifunction elastic warp-knitted fabric was fabricated with the use of crisscross-section polyester/ bamboo charcoal polyester blending yarns on the fabric back and metal composite yarns/viscose blending yarns on the front face of the elastic warp-knitted structure. Rubber thread is fabricated into the fabric to increase the elastic property in walewise direction. The fabric's moisture management property, which influences the sensation of heat-moisture comfort, was assessed in terms of three factors, namely, drying rate, WVT, and water absorbency behavior. In addition, the antibacterial activity and stretch properties of the produced elastic warpknitted fabric were also investigated. 


\section{Experimental}

\subsection{Materials}

Two types of blending yarns (multiply yarns) A and B were used to fabricate the elastic warp-knitted fabric as the incorporated weft yarn for the back and front face of the knitted fabric, respectively. The components of blending yarn $A$ included bamboo charcoal polyester (BC-PET) (provided by Hua Mao Co., Ltd., Taiwan) and crisscross-section polyester filament (CSP) (provided by Everest Textile Co., Ltd., Taiwan) with different blending ratios. Blending yarn $\mathrm{B}$ was composed of Bamboo charcoal polyester (BC-PET)/antibacterial nylon (AN)/stainless steel wire (SSW) metal composite yarns and viscose yarns with fixed proportion. The AN yarn was provided by Industrial technology research institute, Taiwan. The BCPET/AN/SSW metal composite yarns were produced on a hollow spindle spinning machine with the use of AN and BCPET around a SSW in the Z-direction and S-direction with 950 TPM, respectively. BC-PET was included in BC-PET/AN/SSW metal composite yarns was to avoid the direct exposure to the antibacterial agent on the fabric surface. The SSW (provided by King's Metal Fiber Technology Co., Ltd., Taiwan) in the metal composite yarns provided antistatic and electromagnetic shielding property. The details of the yarns used in this study are presented in Table 1.

\subsection{Fabric preparation}

A crochet machine (Dah Heer Industrial Co., Ltd., Taiwan) was used to manufacture the elastic warp-knitted fabric specimens. When the PET warp yarns were drawn into the needle bare and looped around the rubber thread, blending yarns $A$ and $B$ were inserted into the knitted fabric on the top and below of the rubber thread. In this study, the hybrid yarn $A$ (multiply yarn) was prepared on a double-wire machine using 150D/144F BC-PET and $75 \mathrm{D} / 48 \mathrm{~F}$ CSP filaments with different blended proportions as shown in Table 2. Hybrid yarn B (multiply yarn) was also prepared on a double-wire machine using 378D BC-PET/AN/ SSW metal composite yarns (wrapped yarn) and 166D viscose staple fibre spun yarns at a fixed 1:2 blended proportion. For the produced elastic warp-knitted fabrics, hybrid yarns $A$ and $B$ were both used as incorporated weft yarns in this type of warpknitted fabrics. The schematic of the crochet machine used to produce elastic warp-knitted fabric was shown in Figure 1. In this study, we defined the side with hybrid $A$ and $B$ as the back and front face of the elastic warp-knitted fabric, respectively (Figure 2). The main purpose of viscose in blending yarn B was to improve the water absorbency and WVT ability of knitted fabrics. In this study, six types of multifunctional EM shielding fabrics $\mathrm{K}-\mathrm{X}$ series (K1, K2, K3, K4, K5, K6) were produced with varying blended proportions of hybrid yarn $A$. The detailed characteristics of the produced elastic warp-knitted fabric are presented in Table 2.

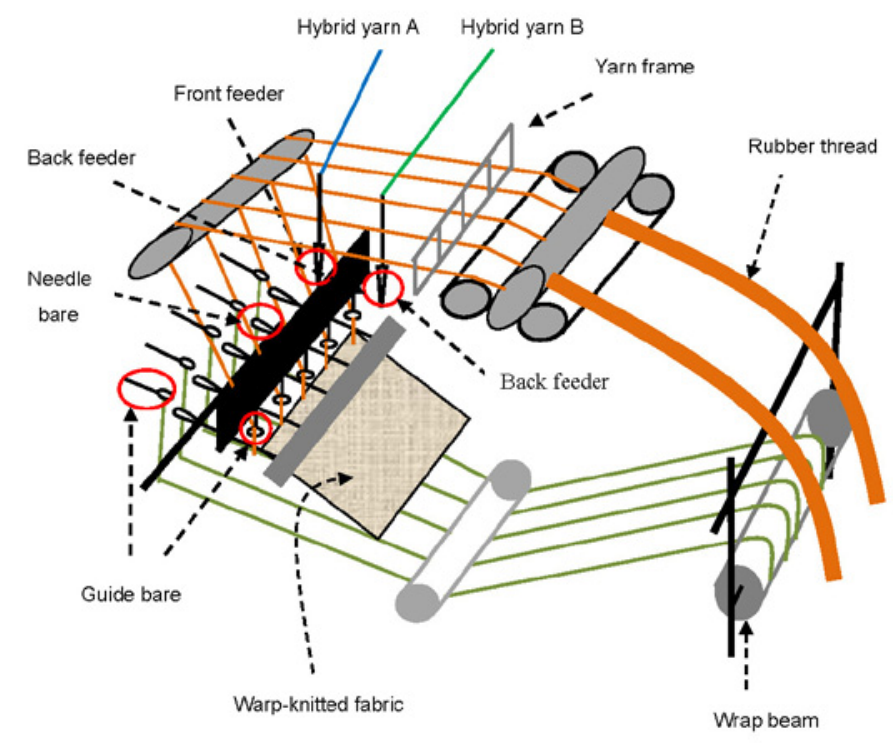

Figure 1. Schematic of the crochet machine used to manufacture elastic warp-knitted fabrics

Table 1. Details of the yarns used in this study

\begin{tabular}{|c|c|c|c|c|}
\hline Yarn type & Linear density (dtex) & Diameter $(\mathbf{m m})$ & Tenacity (cN/dtex) & Elongation (\%) \\
\hline CSP & 83.3 & 0.154 & 3.572 & 36.1 \\
\hline AN & 166.7 & 0.286 & 3.851 & 26.9 \\
\hline BC-PET & 166.7 & 0.301 & 3.520 & 16.24 \\
\hline SSW & 154.4 & 0.050 & 0.985 & 33.7 \\
\hline BC-PET/AN/SSW & 521.1 & 0.351 & 2.161 & 29.1 \\
\hline
\end{tabular}

Note: BC-PET: bamboo polyester filament; CSP: crisscross-section polyester filaments; PET: polyester filament; SSW: stainless steel wire; BCPET/AN/SSW: metal composite yarns. 


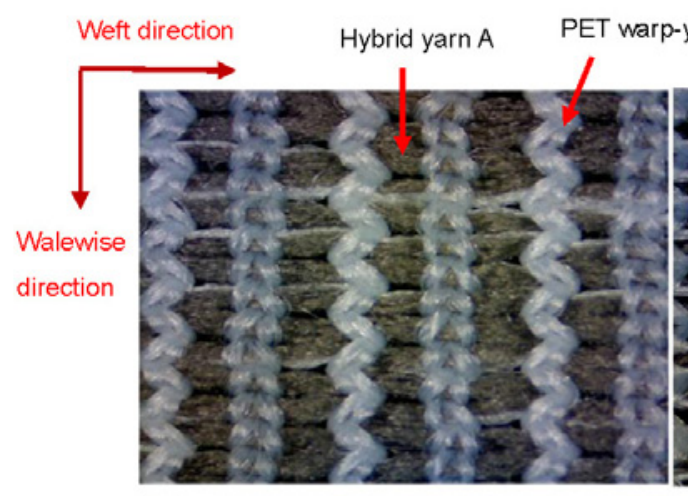

(a)

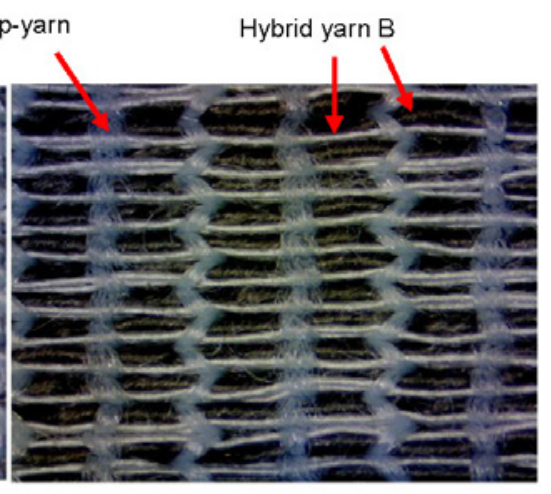

(b)

Figure 2. Microscopic images of back face (a) and front face (b) of produced elastic warp-knitted fabric

Table 2. The basic structure parameters of elastic warp-knitted fabric samples

\begin{tabular}{|c|c|c|c|c|c|c|}
\hline \multirow{2}{*}{$\begin{array}{l}\text { Sample } \\
\text { cold }\end{array}$} & \multicolumn{2}{|c|}{ Composition of multiple hybrid yarns } & \multirow{2}{*}{$\begin{array}{c}\text { Wale } \\
\text { density } \\
\text { Wales } / \mathrm{cm}\end{array}$} & \multirow{2}{*}{$\begin{array}{c}\text { Weft } \\
\text { density } \\
\text { Picks/cm }\end{array}$} & \multicolumn{2}{|c|}{ Other properties } \\
\hline & $\begin{array}{c}\text { Hybrid yarn A } \\
\text { Proportion (600D) }\end{array}$ & $\begin{array}{c}\text { Hybrid yarn B } \\
\text { Proportion (580D) }\end{array}$ & & & $\begin{array}{l}\text { Weight } \\
\left(\mathbf{g} / \mathbf{c m}^{2}\right)\end{array}$ & $\begin{array}{l}\text { Thickness } \\
\text { (mm) }\end{array}$ \\
\hline K1 & 4BC-PET & BC-PET/AN/SSW: 2VY & 6.1 & 20.5 & 51.4 & 27.5 \\
\hline K2 & 3BC-PET:2CSP & BC-PET/AN/SSW: 2 VY & 6.2 & 20.1 & 52.2 & 28.0 \\
\hline K3 & 2BC-PET:4CSP & BC-PET/AN/SSW: 2VY & 6.1 & 20.6 & 51.1 & 27.6 \\
\hline K4 & 1BC-PET:6CSP & BC-PET/AN/SSW: 2 VY & 6.3 & 20.1 & 49.8 & 28.1 \\
\hline K5 & $8 \mathrm{CSP}$ & BC-PET/AN/SSW: 2 VY & 6.0 & 20.4 & 49.9 & 27.8 \\
\hline K6 & 4PET & 4PET & 6.2 & 20.7 & 51.8 & 20.3 \\
\hline
\end{tabular}

Note: BC-PET: bamboo polyester filament; CSP: crisscross-section polyester filaments; VY: viscose filament; PET: polyester filament; SSW: stainless steel wire; BC-PET/AN/SSW: metal composite yarns

\subsection{Test methods}

\section{Water vapour transmission testing}

Water cup method was used to assess the WVT rate in accordance with ASTM E96/E96M-05. A round cup was filled with distilled water up to $3 / 4$ level. Afterward, a sample was set onto the round cup and sealed with a washer while exposing only a sample area of $30 \mathrm{~cm}^{2}$. Finally, the test apparatus was placed under a specified atmospheric condition $\left(20 \pm 2{ }^{\circ} \mathrm{C}\right.$, $65 \pm 5 \%$ relative humidity) for $24 \mathrm{~h}$. The WVT of the fabric was calculated by using Equation (1).

$W V T=G / T A$

where $W V T$ is the rate of WVT $\left(\mathrm{g} / 24 \mathrm{~h} . \mathrm{m}^{2}\right), G$ is the weight change of the test cup $(\mathrm{g}), T$ is the time during which $G$ occurred (h), and $A$ is the test area $\left(\mathrm{m}^{2}\right)$.

\section{Drying ability testing}

Drying ability was evaluated on the basis of the water evaporation rate (WER) of the fabric in accordance with GB/T 21655.1-2008.The specimen was cut into at least100 $\mathrm{mm} \times$
$100 \mathrm{~mm}$ squares and placed on an experimental platform. Approximately $0.2 \mathrm{~g}$ of distilled water was dropped on the surface of the sample, which was weighted immediately. Afterward, the specimen was hanged under standard atmospheric condition ( $20 \pm 2{ }^{\circ} \mathrm{C}, 65 \pm 2 \%$ relative humidity). The change in water was continuously recorded at regular intervals until the change rate was lower than $1 \%$. Finally, the WER was calculated by using Equation (2).

$$
\text { WER }(\%)=\frac{\Delta \mathrm{m}}{\text { qualitly of dropped water }} \times 100 \%
$$

where WER is the water evaporation rate, and $\Delta \mathrm{m}$ is the change in water weight, $\mathrm{g}$.

\section{Water absorbency testing}

The method proposed by Mukhopadhyay et al. [13] was adopted in measuring the water absorbency of the elastic knitted fabric. The samples were cut into sample sizes of 10 $\mathrm{cm} \times 10 \mathrm{~cm}$ and the weight of each sample was recorded. The samples were dipped in water for a certain time, taken out, and then kept on a sponge sheet for $30 \mathrm{~min}$ to remove the 
excess surface water. Subsequently, the water absorbency of the fabric was evaluated by using Equation (3).

$$
A=\frac{w_{2}-w_{1}}{w_{1}} \times 100
$$

where $A$ is the water adsorption (\%), W1 represents the quality of the original specimen $(\mathrm{g})$, and $W 2$ represents the specimen soaked in distilled water $(\mathrm{g})$.

\section{Assessment of antibacterial activity}

A qualitative method based on AATCC90-2011 was used to assess the antibacterial activity of the produced knitted fabrics. Two test strains, Escherichia coli (E. coli) and Staphylococcus aureus (S. aureus) were used in this study. All of the plates were observed for a zone of inhibition after $24 \mathrm{~h}$ of incubation.

\section{Results and discussion}

\subsection{Water vapour transmission testing results}

When the produced elastic warp-knitted fabrics were worn during summer or during sports activities, some of the water vapour should be transported from the skin to the external environment through the air gap in the fabric. If a garment has a high WVT, water vapour is quickly transported out and the humidity on the surface of the skin is decreased, thereby increasing comfort. Figure 3 shows the WVT values of the manufactured elastic warp-knitted fabric K-X series. Results showed that increasing the blend proportion of BC-PET yarns in blending yarn A slightly increases WVT. This slight increase in WVT is attributed to the presence of bamboo charcoal in the BC-PET yarns, which facilitates good humidity adjustment. The bamboo charcoal could absorb the water vapour from regions of high concentration to regions of low concentration. Thus, fabric K1 displayed the highest WVT because it had the highest BC-PET content. Although the CSP fibre had a large superficial area because of the grooves on the surface, CSP fibre had low water vapour adsorption and release ability because of its chemical structure.

\subsection{Water absorption rate testing results}

As shown in Figure 4, the water absorbency percentage of fabric $\mathrm{K} 5$ was the highest, whereas that of fabric K1 was the lowest. In general, fabric thickness and porosity significantly influence water absorbency because higher fabric thickness could provide more space for water entrapment. However, the elastic warp-knitted fabrics have nearly the same fabric thickness and weight (Table 2). Thus, these findings were mainly attributed to the abundant gaps among fibers in the CSP or the water entrapment ability as a result of the presence of grooves on the surface of the CSP. Therefore, fabric K5 showed the highest water absorption rate because it had the highest CSP content among the elastic warp-knitted fabrics. Proper water absorbency could quickly absorb perspiration from human body, which is beneficial in decreasing air humidity around the skin and the fabric inner.

\subsection{Drying rate testing results}

Fabric drying was assessed in terms of perspiration evaporation under standard atmospheric conditions. Evaporation of water or sweat is the primary mechanism underlying the drying of fabric. Water evaporation provides a cool feeling because part of the heat energy is taken away following evaporation, suggesting that drying rate has an important role in heat-moisture comfort [14-16]. As shown in Figure 5, fabric K5 showed the highest WER, whereas fabric K1 displayed the lowest WER. These results can be attributed to the groove on the surface of CSP, which provided higher capillary pressure than common polyester or BC-PET. Therefore, with increasing CSP content in the elastic warp-knitted fabrics, the fabric is expected to have better water transfer and a larger diffusion area. Notably, WER was not high for all of the fabric F-X series, possibly because of the high fabric density, weight, which resulted from the incorporation of rubber thread in the manufactured fabrics.

\subsection{Antibacterial activity evaluation of the elastic warp- knitted fabric}

After considering the drying rate, WER, and water absorbency, we chose fabric $\mathrm{K} 5$ as the functional textile to investigate the antibacterial activity of the elastic fabric. Qualitative assessment

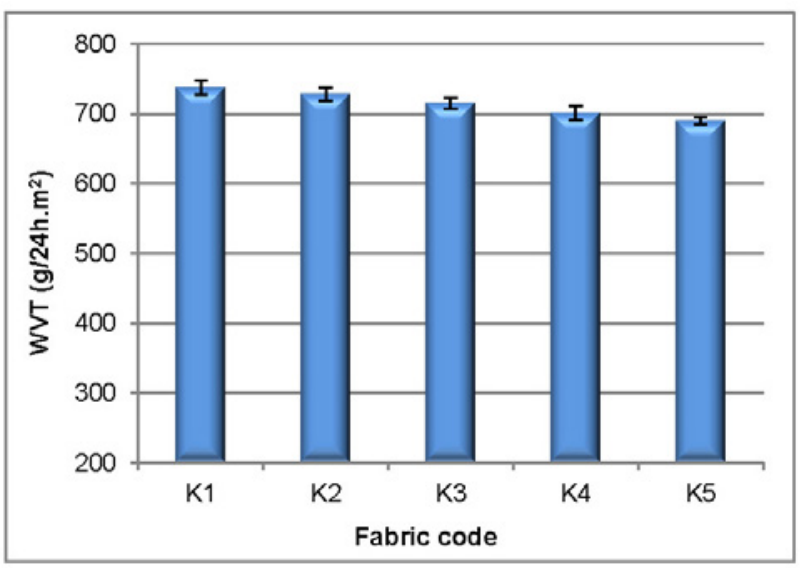

Figure 3. WVT ability of elastic warp-knitted fabric F-X series

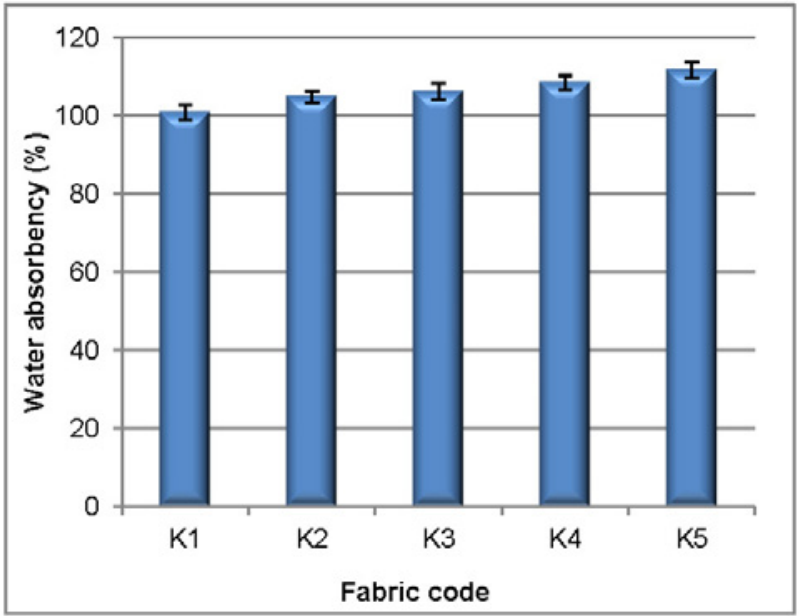

Figure 4. Water absorption rate of elastic warp-knitted fabrics F-X series 
method was used to evaluate the antibacterial activity of the fabric by observing the inhibition zones on the plates after $24 \mathrm{~h}$ of incubation with two test strains, namely, E. coli and $S$. aureus (Figure 6). Figure 6 showed that fabric K5 displayed great antibacterial activity against both types of bacteria as demonstrated by the inhibition zone on the front face of the fabric. In comparison with PET knitted fabric, the antibacterial activity of fabric $\mathrm{K} 5$ is caused by the presence of antibacterial nylon in BC-PET/AN/SSW metal composite yarns which affects the DNA molecules of the bacteria and reduces their capability to replicate and transfer, thus causing bacterial death and the generation of an inhibition zone [17-18].

The back face of the fabric showed no inhibition zone, which is expected because the incorporated CSP in the back of the fabric did not have any antibacterial activity. Fabric serves as a media between the human body and the environment. Thus, we hope only the surface of the garment has antibacterial activity while the back face of the garment did not have antibacterial activity to protect the skin from the possible harmful effects of the antibacterial agent. Therefore, the produced elastic warpknitted fabric was an ideal antibacterial protective fabric.

\subsection{Stress-extension curves of the elastic warp-knitted} fabric

The fabric should have good extension performance when subjected to stretch by ordinary body movement or sports activity [19]. Higher extension and lower stress values of the fabric would provide a good pressure comfort property for wearers. In this work, we chose fabric K5 as a representative to assess the stress-extension of the elastic warp-knitted fabric in the weft and walewise direction, respectively. As shown in Figure 7, fabric K5 showed better stretching performance in the walewise direction than in the weft direction. The breaking elongation of the elastic fabric $\mathrm{K} 5$ was close to 300 and $20 \%$ in the walewise and weft direction, respectively, mainly because the rubber thread was only present in walewise direction. Moreover, the fabric stress value was low $\left(0.02 \mathrm{~N} / \mathrm{mm}^{2}\right)$ and the extension grew more rapidly than the stress when the extension

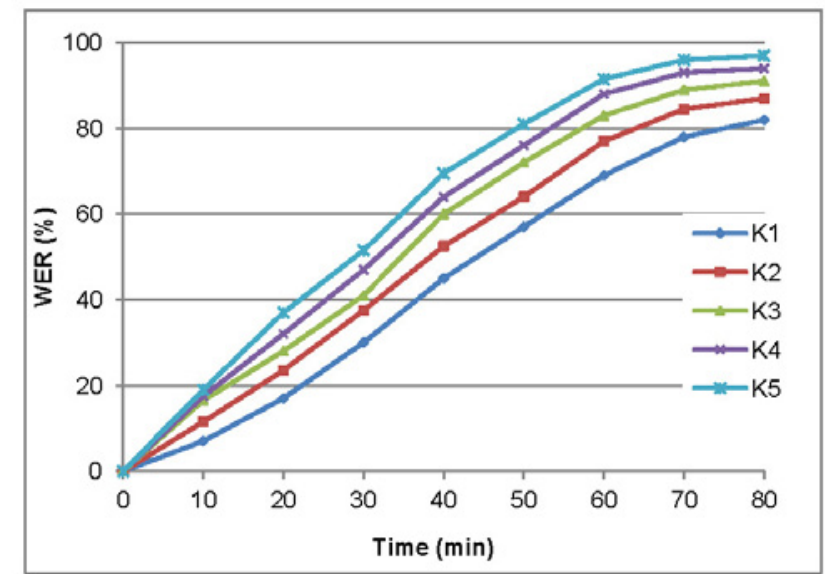

Figure 5. WER cures for elastic warp-knitted fabric K-X series.

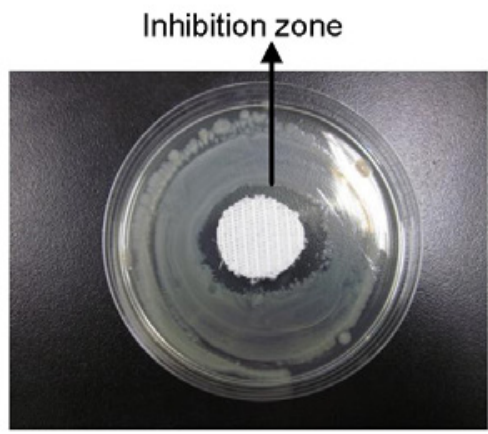

Front face

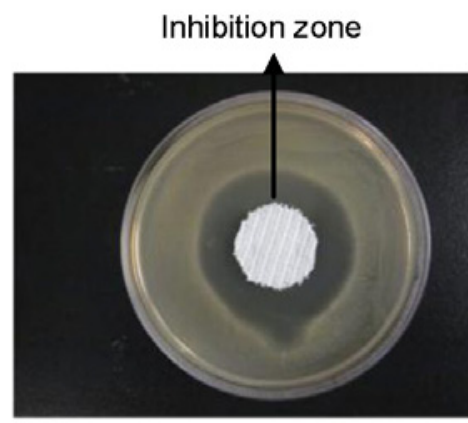

Front face

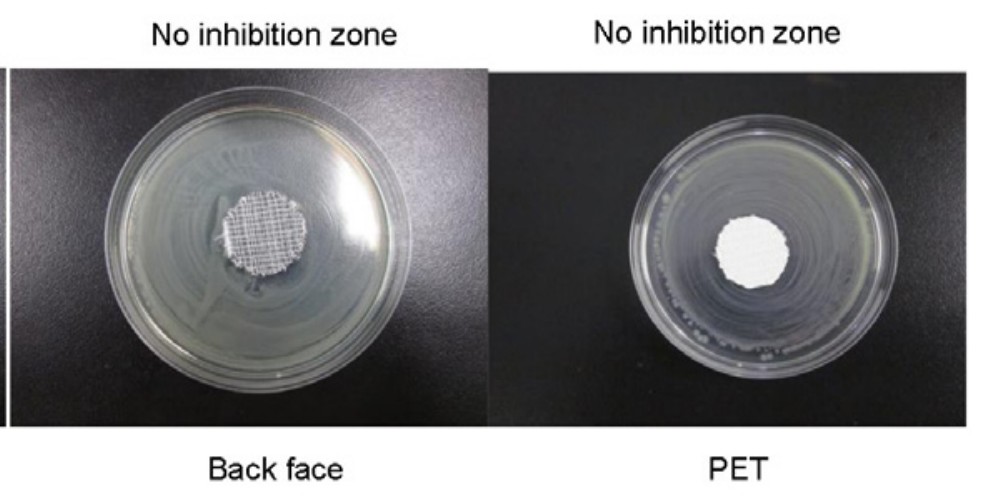

(a) E. coli

No inhibition zone

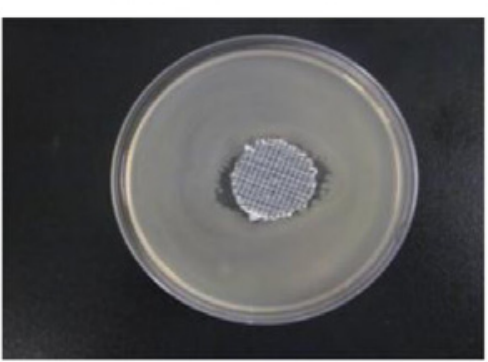

Back face

PET

(b) S. aureus

Figure 6. The zone of inhibition for back and front face of fabric K5. 


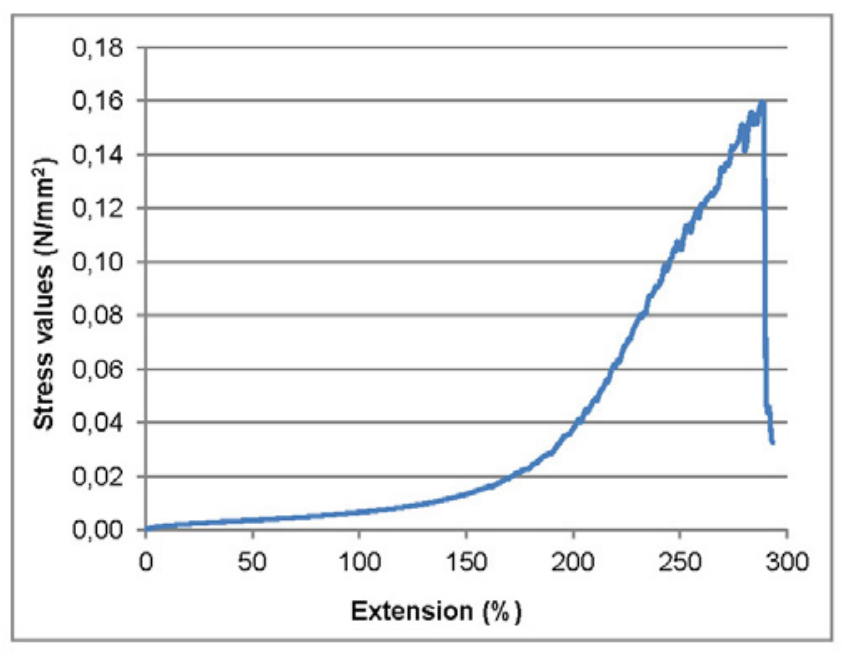

(a)

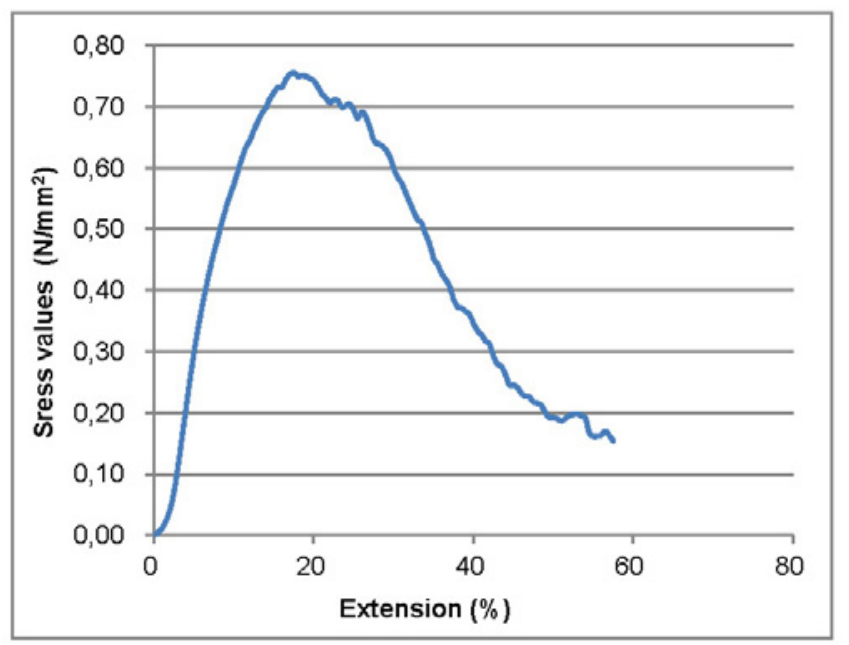

(b)

Figure 7. Stress-extension curves for the fabric $\mathrm{K} 5$ (a) in the walewise direction; (b) in the weft direction.

was in the range of 0 to $170 \%$ in walewise direction. Thus, the garment is expected to provide low resistance to the human body when the garment is stretched during sports activity.

\section{Conclusions}

In this study, a kind of multifunction elastic warp-knitted fabric was produced on a crochet machine with functional blending yarns $\mathrm{A}$ and $\mathrm{B}$. The WVT, drying rate, water absorbency, antibacterial activity, and stretch properties of the produced knitted fabrics were investigated and the following conclusions were drawn:

1. With increasing BC-PET content in the fabric, WVT values also increased because of the water vapour absorption properties of the bamboo charcoal component in the BC-PET.

2. Fabric $\mathrm{K} 5$ displayed the highest drying rate and water absorbency ability among all of the fabrics in the $\mathrm{K}-\mathrm{X}$ series because of the grooves on the surface of the CSP fibre.
3. Fabric K5 showed satisfactory antibacterial activity on the front face of the knitted fabric, whereas the back face of the fabric showed no antibacterial activity.

4. The breaking elongation of the elastic warp-knitted fabric displayed better elongation and lower stress values in the walewise direction than in the weft direction. Further study will be carried to investigate the thermal comfort property of the produced elastic warp-knitted fabrics.

\section{Acknowledgements}

The authors are grateful to the Laboratory of Fiber Application and Manufacturing, Feng Chia University, for providing research materials, laboratory equipment and financial support under Contract NSC-103-2221-E-035-028. This work was also supported by the Chinese Nature Science Foundation [No.51343002], Key Discipline Project of Liaoning Province Universities [No.2012310], and Project of Functional Textile Materials Laboratory of Eastern Liaoning University.

\section{References}

1. Wang, Y.R., Zhang, P.H., Zhang, Y.P. (2014). Experimental investigation the dynamic pressure attenuation of elastic fabric for compression garment. Textile Res J, 84(6), 572582.

2. Filgueiras, A., Fangueiro, R., Soutinho, F. (2009). Drape behavior of functional knitted fabrics for sport clothing. Indian J Fibre Text Res, 34, 64-68.

3. Komisarczyk, A., Dziworska, G., Krucinska, I., Michalak, M., Strzembosz, W., Kaflak, A., Kaluza, M. (2013). Visualisation of liquid flow phenomena in textiles applied as a wound dressing. Autes Res J, 13(4), 141-149.

4. Gorjanc, D.S., Dimitrovski, K., Bizjak, M. (2012). Thermal and water vapor resistance of the elastic and conventional cotton fabrics. Text Res J, 82(4), 1498-1506.

5. Onofrei, E., Rocha, A.M., Catarino, A. (2012). Investigation the effect of moisture on the thermal comfort properties of functional elastic fabrics. J Ind Text, 42(1), 34-51.

6. Fukazawa, T., Havenith, G. (2009). Differences in comfort perception in relation tolocal and whole body skin wettedness. Eur J Appl Physiol, 106(1), 15-24.

7. Laing, R.M., Wilson, C.A., Gore, S.E., Carr, D.J. (2007). Determining the drying time of apparel fabrics. Text Res $J$, 77(8), 583-590.

8. Fourt, L., Sookne, A.M., Frishman, D., Milton, H. (1951). The rate of drying of fabrics. Text Res J, 21(1), 26-33.

9. Fangueiro, R., Filgueiras, A., Soutinho, F., Xie, M.D. (2010). Wicking behavior and drying capability of functional knitted Fabrics. Text Res J, 80(15), 1522-1530.

10. Duru, S.C., Candan, C. (2013). Effect of repeated laundering on wicking and drying properties of fabrics of seamless garments. Text Res J, 83 (6), 591-605.

11. Bedek, G., Salaun. F., Martinkovska, Z., Devaux, E., Dupont, D. (2011). Evaluation of thermal and moisture management properties on knitted fabrics and comparison with a physiological mode in warm conditions. Applied Ergonomics, 42(6), 792-800. 
12. Senthilkumar, M., Anbumani, N. (2011). Dynamics of elastic knitted fabrics for sports wear. J Ind Text, 41(1), 13-23.

13. Mukhopadhyay, A., Ishtiaque, S.M., Uttam, D. (2011). Impact of structural variations in hollow yarn on heat and moisture transport properties of fabrics. J Text Inst, 102 (8), 700-712.

14. Bivainyte, A., Mikucioniene, D. (2011). Investigation on the air and water vapor permeability of double-layered weft knitted fabrics. Fibres Textiles East Eur, 19(3), 69-73.

15. Fohr, J.P., Couton, D., Treguier, G. (2002). Dynamic heat and water transfer through layered fabrics. Textile Res $\mathrm{J}$, 72(1), 1-12.

16. Prahsarn, C., Barker, R.L., Gupta, B.S. (2005). Moisture vapor transport behavior of polyester knit fabrics. Textile Res J, 75(4), 346-351.

17. Bagherzadeh, R., Montazer, M., Latifi, M., Sheikhzadeh, M., Sattari, M. (2007).

Evaluation of comfort properties of polyester knitted spacer fabrics finished with water repellent and antimicrobial Agents. Fiber Polym, 8(3), 386-392.

18. Wang, X., Yin, Y.J., Wang, C.X. (2010). New approach to impart antibacterial effect and improve ink jet printing properties with modified $\mathrm{SiO}_{2}$ sols cationic biocides. Colloid Surf A-Physicochem Eng Asp, 361(1-3), 51-55.

19. Mukhopadhyay, A., Sharma, I.C., Mohanty, A. (2003). Impact of lycra filament on extension and recovery characteristics of cotton knitted fabric, Indian J Fibre Text Res, 28(4), 423-430. 